\title{
Energy Management Strategy for Oscillating Drivetrains Equipped with an Electric Variable Transmission
}

\author{
Srajan Goyal \\ DecisionS \\ Flanders Make \\ Lommel, Belgium \\ srajan.goyal@flandersmake.be
}

\author{
Florian Verbelen \\ Department of Electrical Energy, Metals, \\ Mechanical Constructions and Systems, Ghent University \\ Ghent, Belgium \\ florian.verbelen@ugent.be
}

\author{
Ahmed A-E. Abdallh \\ MotionS \\ Flanders Make \\ Leuven, Belgium \\ ahmed.abdallh@flandersmake.be
}

\author{
Kurt Stockman \\ Department of Electrical Energy, Metals, \\ Mechanical Constructions and Systems, Ghent University \\ Ghent, Belgium \\ kurt.stockman@ugent.be
}

\author{
Peter Sergeant \\ Department of Electrical Energy, Metals, \\ Mechanical Constructions and Systems, Ghent University \\ Ghent, Belgium \\ peter.sergeant@ugent.be
}

\begin{abstract}
In this study the dynamic capability of the Electric Variable Transmission (EVT) is presented based on the tracking of a highly dynamic oscillating load. The targeted applications are 3-phase grid connected machines with periodic motions at high frequencies $(>5) \mathbf{H z}$, which result in a high alternating to average power ratio $(>5)$. The overall consumed grid energy is minimized by a high-level non-parametric cascaded control to recuperate the oscillating load energy in a mechanical energy storage component. Here in this paper, this oscillating energy is stored in the inner rotor of the EVT, thereby making EVT an energy storing device in itself. The drivetrain containing an EVT is also shown to have a good load speed tracking performance with the maximum error of $\pm 1 \%$.

Index Terms-Cascaded control, dynamic modeling, electric variable transmission, energy minimization, oscillating drivetrains.
\end{abstract}

\section{INTRODUCTION}

An Electric Variable Transmission (EVT), also called dual mechanical port electric machine, is a competitive alternative power split device that combines the functionality of two electrical machines and a planetary gear set into one single electromechanical device [1]. It is also shown in the same paper that an EVT can significantly reduce the fuel consumption in a Hybrid Electric Vehicle (HEV). However, due to the relatively modest dynamic requirements in automotive applications, the dynamic capabilities of the EVT are not fully utilized. To the best of authors' knowledge, the dynamic behavior of the EVT has not been fully investigated yet. This aspect has been covered in this paper by utilizing the dynamic behavior of the considered EVT by driving high frequency oscillating loads.

One of the most important grid connected industrial dynamic applications are production machines with fast reciprocating loads $(>5) \mathrm{Hz}$, e.g. weaving looms and plate punching machines. The subsequent acceleration and deceleration gives rise to a reciprocating energy flow which is converted back and forth between the electrical and mechanical components. Compared to the slow energy storage of elevators and cranes, these kind of applications exhibit a much higher power to energy ratio. In order to recuperate this energy, either a fully controlled Active Front End (AFE) or a Passive Front End (PFE) with an electric energy storage device, e.g. capacitor bank, can be used [2]. Another way to recover this reciprocating energy is by using a mechanical flywheel, attached to the shaft of a traditional motor, where the recovered energy can be reciprocated back during acceleration of the load. The main issue with this solution is that the flywheel needs to be accelerated or decelerated during start up and shut down. As start-up times are crucial in industrial machines (delays are not allowed or should be minimized), higher torque values during start-up are required. This often results in the necessity of a larger electric machine, i.e. oversized motor, to drive the application. As a consequence, this electric motor will only be used efficiently during start-up. For the remaining normal operation, the motor is used in partial load which is highly inefficient. Alternatively, an EVT can be used instead of the motor. Thanks to the EVT unique configuration, the flywheel is mechanically disconnected from the load, hence fast start-up and stop processes are possible. Also, higher speed variations are possible with a flywheel providing more flexibility in terms of energy transfer.

In order to optimize the energy drawn from the grid, the overall system needs to be controlled, via a proper energy management strategy, in concurrent with the EVT current control. The energy management problem for dynamic machines is non-convex, highly nonlinear mainly due to the nonlinear behavior of various electric components and electro-magnetic 
coupling between them. The system-level control strategy has to deal with highly dynamic load disturbances, interactions within the system between different components, while also satisfying system constraints.

Energy management of an EVT based system has been explored to a good extent in the literature [1], [3]-[5]. However studies on its applicability in real world systems are relatively scarce. Energy management of an HEV equipped with an EVT has been approached using dynamic programming in [3] for an optimal power split and overall cost minimization, and based on fuzzy logic global power management strategy in [4]. In [6], the authors have used a static EVT model to investigate the impact of the EVT in HEVs on the fuel consumption. The paper shows that a significant reduction in fuel consumption is possible compared to series and parallel powersplit topologies. However, the dynamic capabilities of an EVT are not at all utilized in the HEV applications due to high time constants of the other components in drivetrain, e.g. internal combustion engine, as well as the related drive cycle. In context of dynamic machines, EVT has been used in dual power flow wind energy conversion systems (DPFWECS) in [7], where an observer based on model reference adaptive system (MRAS) has been combined with sliding mode control to control inner and outer rotors of the EVT. However, energy is stored/supplied directly to/from the grid requiring an additional electrical energy storage system. Most importantly, the dynamic behavior of EVT is not fully realized because of low frequency load application.

The aim of this paper is to demonstrate how proper energy management can result in efficient energy recuperation in an industrial machine equipped with an EVT driving high frequency loads. As far as we know, this has not been presented before. The objective is to realize the dynamic capabilities of an EVT via a good load tracking performance along with minimization of overall energy costs associated with the grid supply. In this paper, only the energy cost has been considered. Installation cost has been disregarded because the EVT is not yet available in mass production.

In the subsequent section II, an overview of the system is given which brings more insight to the EVT and other components. The developed energy management approach is discussed along with the control challenges faced in section III, followed by the use case in section IV. In section V, corresponding results and discussion are presented and finally, conclusions of the paper are drawn in section VI.

\section{System OVERVIEW}

The schematic of the considered drivetrain is shown in Fig. 1, where the EVT is used as a power split device between different energy sources. The outer rotor is connected to the oscillating load, whereas the inner rotor is connected to a mechanical flywheel which acts as energy storage ${ }^{1}$. The DC

\footnotetext{
${ }^{1}$ Please notice that this figure shows the generic system including an additional energy storage device. It is worth mentioning that the energy can be stored in the inner rotor of the EVT itself, which acts as a flywheel, as described in this paper.
}

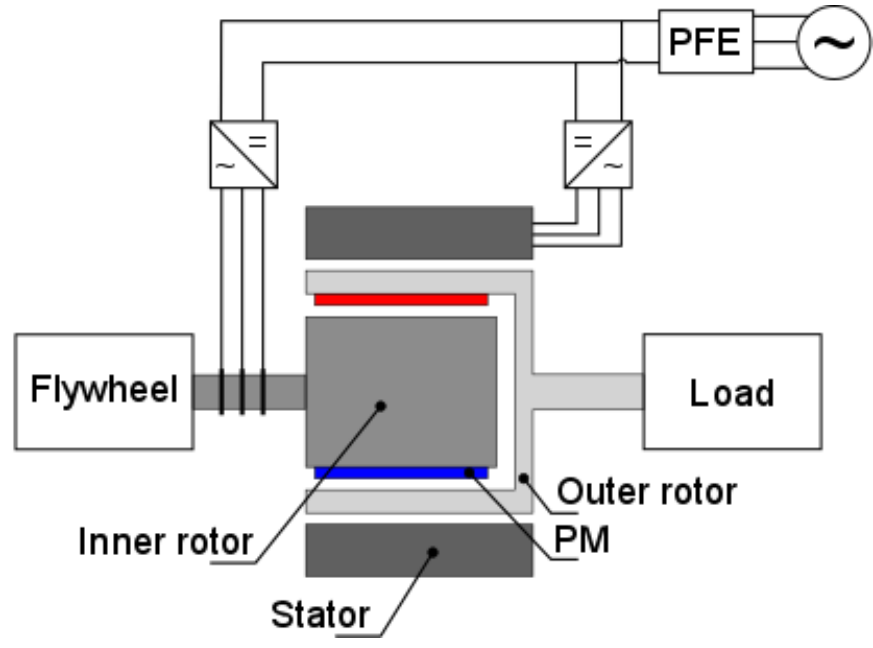

Fig. 1. Schematic diagram of the considered drivetrain with EVT.

(direct current) bus is connected to a three-phase, $400 \mathrm{~V}$ power grid via an uncontrolled three phase rectifier circuit, which is called later a PFE due to which, energy can not be supplied back to the grid. It implies that the oscillating energy needs to be solely stored in the flywheel, whereas the grid needs to feed only the non-oscillating active energy. In this section, we will illustrate briefly the system components in section II-A, followed by the formulation of the problem in section II-B.

\section{A. System Components}

The considered EVT is a complex device in which both the stator and inner rotor consist of a distributed three-phase winding. On the outer rotor, a single layer of permanent magnets is combined with a DC-field winding to regulate the stator flux linkage. More details concerning this unique feature and the dependency of the DC-field current on torque generation can be found in [8], [9].

To reduce the subset of currents which determine the generated torque, the $d-q$ reference frame is chosen (related to the outer rotor). The torque which is generated by the stator $T_{s}$ and the inner rotor $T_{r 1}$ can now be determined based on (1) and (2), respectively. Because the sum of the generated torque values should be zero, it is possible to obtain the torque on the outer rotor based on (3).

$$
\begin{gathered}
T_{s}=\frac{3}{2} N_{p}\left(\Psi_{s q} I_{s d}-\Psi_{s d} I_{s q}\right) \\
T_{r 1}=\frac{3}{2} N_{p}\left(\Psi_{r 1 q} I_{r 1 d}-\Psi_{r 1 d} I_{r 1 q}\right) \\
T_{r 2}=-T_{s}-T_{r 1}
\end{gathered}
$$

In the previously defined equations the following parameters are used: $N_{p}$ is the number of pole pairs, $\Psi_{s q}$ and $\Psi_{s d}$ are the stator flux in the $q$-axis and $d$-axis respectively, $\Psi_{r 1 q}$ and $\Psi_{r 1 d}$ are the inner rotor flux in the $q$-axis and $d$-axis respectively, $I_{s q}$ and $I_{s d}$ are the stator current in the $q$-axis and $d$-axis 
respectively, $I_{r 1 q}$ and $I_{r 1 d}$ are the inner rotor current in the $q$-axis and $d$-axis respectively. Note that the current in the DC-field winding $I_{r 2 d}$ is not visible in these equations as it merely influences the fluxes. In this paper, the subscripts $r_{1}$ and $r_{2}$ imply the corresponding signal on inner and outer rotor respectively.

The fluxes which are defined in (1) and (2) are calculated based on a finite element model. As solving this model requires time and high computational effort, the calculations are done beforehand and the results are stored in look-up tables (LUTs) which are consulted during simulation.

When taking a closer look at both (1) and (2), it is convenient to see that an infinite number of current combinations results in the same generated torque. However, only one set of currents will result in the lowest amount of losses (sum of copper and iron losses). A method to determine this optimal subset of currents as function of the operating point (torque and speed of both shafts) has been elaborated in [9]. By implementing this method in the model, the losses of the EVT are minimized at a chosen operating point. An evaluation of the losses (copper, iron, bearing and inverter losses) and how these are modelled can be found in [10].

In the previous paragraphs the relation between current and torque has been discussed. However, to accurately describe the generated torque of the EVT during dynamic load variations it is important to take the current dynamics into account as these determine how fast torque can be generated. In order to calculate the current which flows through the windings the following equation is used:

$$
V_{i}=R_{i} I_{i}+\frac{d \Psi_{i}}{d t}
$$

in which subscript $i$ indicates the applicability for stator and inner rotor, $V_{i}$ is the voltage applied to the windings, $I_{i}$ is the current which flows through the windings and $\frac{d \Psi_{i}}{d t}$ is the derivative of flux to time. To solve (4), it is rewritten as:

$$
V_{i}=R_{i} I_{i}+\frac{d \Psi_{i}}{d I_{i}} \frac{d I_{i}}{d t}
$$

in which $\frac{d \Psi_{i}}{d I_{i}}$ is the derivative of flux to current which is also calculated based on the finite element model.

Next to the electromagnetic part of the EVT, there are two bi-directional DC/AC converters to control the EVT, where the DC-bus of these converters are connected to the grid via a PFE. It is worth mentioning here that the AFE is not utilized in this system because the energy needs to be recuperated mechanically in the inner rotor of the EVT or in the attached flywheel, which also reduces the overall system costs.

\section{B. Problem Formulation}

The main objective is to minimize the energy cost of the system with EVT by its optimal control over a dynamic load cycle under the boundary operating conditions for the EVT. Torque setpoints of both rotors of the EVT need to be controlled to minimize the energy cost while also satisfying input and output constraints. The problem here is formulated below:

$$
\min _{T r_{1, s}, T r_{2, s}} \int_{0}^{t_{f}} \text { Energy } \operatorname{Cost}(t) d t
$$

subjected to:

$$
\begin{gathered}
200 \leq T_{r 1, s}(t), T_{r 2, s}(t) \leq 200 \mathrm{Nm} \\
\left|T_{r 1, s}(t)+T_{r 2, s}(t)\right| \leq 300 \mathrm{Nm} \\
-6000 \leq \omega_{r 1, r 2}(t) \leq 6000 \mathrm{rpm} \\
\left|\omega_{r 1}(t)-\omega_{r 2}(t)\right| \leq 4000 \mathrm{rpm} \\
450 \leq V_{D C} \leq 700 \mathrm{~V}
\end{gathered}
$$

where $t$ is the time instant and $t_{f}$ is the duration of dynamic load cycle. Energy cost is defined in terms of the cost equivalent of power consumed from the grid. Equations $(7,8)$ define the constraint on input torque setpoints on both rotors. Equations $(9,10)$ are output constraints on the speed of rotors. Equation (11) is a system constraint on voltage of the DC bus for safe operation.

\section{EnERgy MANAGEMEnT Strategy}

Two levels of control are used: on component and system level. The component-level control of the EVT is done by controlling the $d-q$ stator and rotor current components as described in [9]. This present paper deals with the systemlevel control design where the objective is to provide torque set-values of both rotors in order to minimize the energy drawn from the grid, which in turn minimizes the energy cost.

Model-based control of such systems, which are based on multiple high-dimensional LUTs and dynamic non-linearities, is a challenging task since the information on system states is not available. Data driven system identification techniques for control are often cumbersome due to the complexity and lack of optimality. Any operation of the machine outside the input operating region used for system identification would need some extrapolation with high probability of erroneous behaviour. Even the operation within this operating region would depend on the fineness of dynamic input data points and the interpolation would not always give the optimal results due to the presence of non-linearities.

The input-output scheme of the drivetrain topology, shown in Fig. 2, gives an insight on interaction of different components from the perspective of system simulation and control. The signals in green are predetermined parameters and setpoints which are given as inputs to the system. The signals in red are optimized variables which are calculated by system-level control action and the energy management strategy (EMS). The remaining signals in black are derived by component-level control.

The proposed EMS is configured in a non-parametric cascaded control structure, as shown in the same figure, considering predefined constraints mentioned in (7-11). It consists of two distinct controllers: a controller for the inner rotor (or flywheel) speed and a controller for the grid power in the outer and inner loop, respectively. The grid power controller will be the fast controller as the change in grid power is usually 


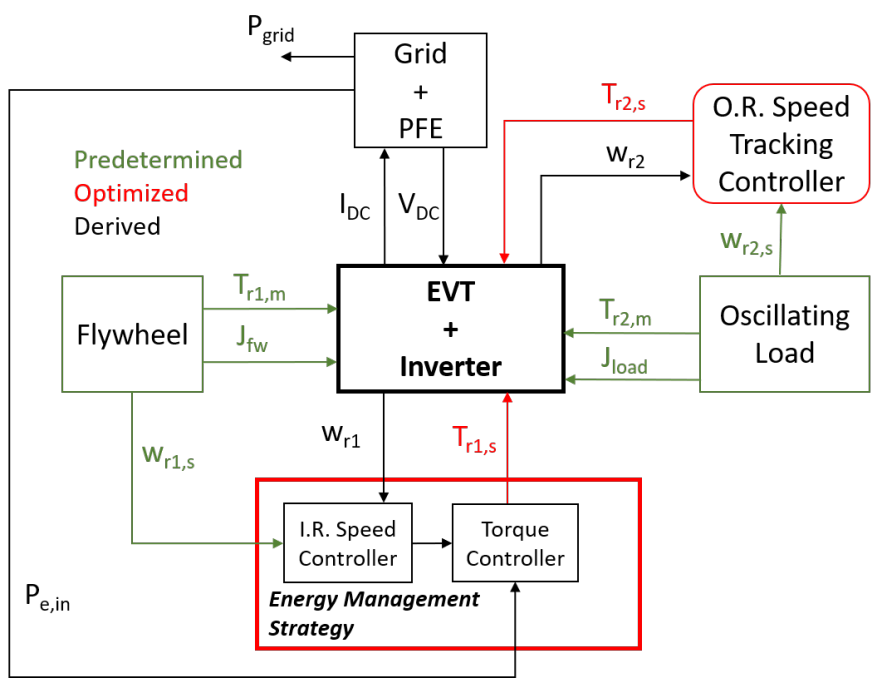

Fig. 2. System level control architecture. I.R. and O.R. stand for inner and outer rotors, respectively.

limited by the response of electrical components (inductance of the windings, switches, etc.). Next is the speed controller which will be slower as it mainly controls a mechanical system, a flywheel or the inner rotor of EVT itself.

By this system-level control action, input electrical power to the EVT, $P_{e, i n}$ is changed such that the inner rotor is controlled around the required setpoint speed, $\omega_{r 1, s}$ throughout the operating range of the dynamic load profile. The output of this control action is the torque setpoint for the flywheel/inner rotor of EVT, which recuperates the maximum energy during deceleration of the load cycle, and the grid power only accounts for the active power and internal EVT losses. A separate speed tracking controller is also implemented on the outer rotor which provides a torque setpoint to control its speed at a desired setpoint, $\omega_{r 2, s}$.

\section{USE CASE}

A physical model is implemented in Simscape ${ }^{\mathrm{TM}}$ for the three-phase supply, PFE and two DC/AC converters, and linked in Simulink ${ }^{\mathrm{TM}}$ with input mechanical load profile and EVT model. The EVT drivetrain model shown in Fig. 1 is simulated via Matlab ${ }^{\mathrm{TM}}$ for an oscillating sinusoidal load torque at a constant speed. The sampling time of system is $0.1 \mathrm{~ms}$. The input mechanical load is given by this equation:

$$
T_{r 2, m}(t)=T_{\text {active }}+T_{\text {alternating }} \cdot \sin (2 \pi f t)
$$

where $f$ is the frequency of sinusoidal load profile and $t$ is the total time. $T_{\text {active }}$ is an offset added due to the DC load. The considered use case, i.e. practical weaving loom application, often has a high alternating to average power ratio. The total mechanical load power is given by the equation:

$$
P_{m, l o a d}=T_{r 2, m} \cdot \omega_{r 2}
$$

TABLE I

EVT SPECIFICATIONS

\begin{tabular}{|l|c|c|}
\hline Parameter & Value & Units \\
\hline Nominal Power & 80 & $\mathrm{kw}$ \\
Inner rotor Inertia $J_{r 1}$ & 0.0598 & $\mathrm{~kg} \cdot \mathrm{m}^{2}$ \\
Outer rotor Inertia $J_{r 2}$ & 0.2608 & $\mathrm{~kg} \cdot \mathrm{m}^{2}$ \\
Flywheel Inertia $J_{f w}$ & 0 & $\mathrm{~kg} \cdot \mathrm{m}^{2}$ \\
Load Inertia $J_{\text {load }}$ & 0 & $\mathrm{~kg} \cdot \mathrm{m}^{2}$ \\
Capacitance (in PFE) & 2 & $\mathrm{mF}$ \\
Line Inductance (in PFE) & 8 & $\mathrm{mH}$ \\
\hline
\end{tabular}

Corresponding to (12), this mechanical load power consists of an active and an alternating part given by:

$$
P_{m, l o a d}=P_{\text {active }}+P_{\text {alternating }}
$$

The speed of inner rotor is to be contained within a certain maximum value based on the maximum speed limits as mentioned in (10). Higher speed means more kinetic energy will be stored in the inertia, according to (15), which results in more stable operation in terms of speed fluctuations as already discussed in section I. If it is desirable to store more energy than what can be achieved at maximum rotor speed limits, an extra inertia in the form of a mechanical flywheel can always be added.

$$
E_{k}=\frac{1}{2} J \cdot \omega^{2}
$$

The dynamics of the outer rotor described by (16), where speed of the outer rotor is based on the electromagnetic torque generated at the outer rotor and the mechanical load torque, $T_{r 2, m}$. An analogue equation is used to calculate the speed on the inner rotor.

$$
\omega_{r 2}(t)=\int \frac{T_{r 2}(t)-T_{r 2, m}(t)}{J_{r 2}^{\prime}} d t
$$

For the outer rotor which is connected to the load, the total inertia is given by (17). An analogue equation is used to calculate the total inertia on the inner rotor.

$$
J_{r 2}^{\prime}=J_{r 2}+J_{l o a d}
$$

Using (16) and (17), higher inertia at the outer rotor would lead to lower dynamics in the speeds and vice-versa which would also affect the start-up and stop time of the machine. However, the system would be much more stable for the same amount of energy recuperation. The design parameters of the drivetrain used to drive the sinusoidal load are mentioned in Table I. A lossless model of the load is considered in this study due to which $J_{\text {load }}=0$. Also, there is no flywheel added to the topology which implies $T_{r 1, m}=0, J_{f w}=0$. Hence, energy will solely be stored and supplied by the inner rotor which acts as a flywheel, thereby making the EVT in itself an energy storage device. The results are shown in the next section.

\section{RESUlts AND Discussion}

The results shown in this section are obtained for a load profile given by (12), with $T_{\text {active }}$ and $T_{\text {alternating }}$ equal to 


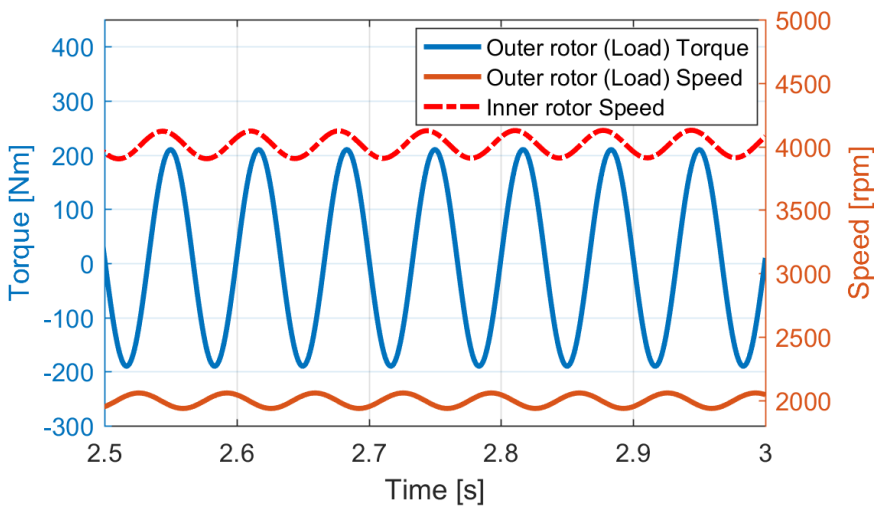

Fig. 3. Oscillating load torque $(15 \mathrm{~Hz})$ and speed tracking

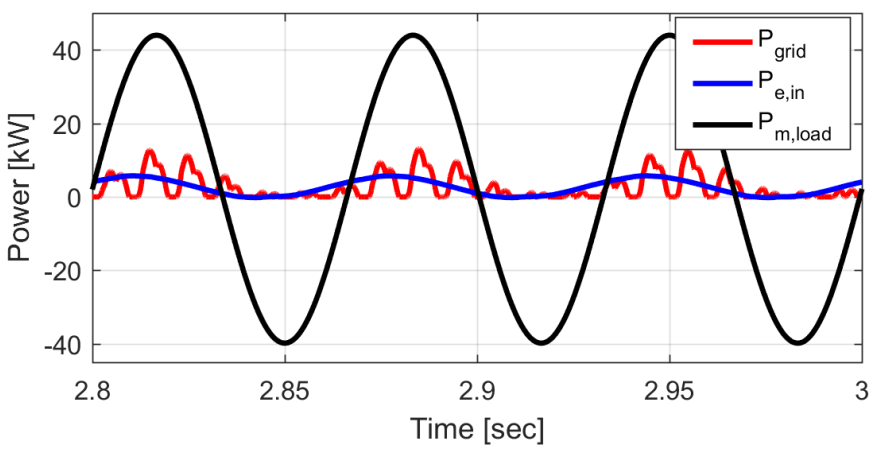

Fig. 4. Comparison of power required to drive the oscillating load with power consumed from grid supply

10 and $200 \mathrm{Nm}$ (i.e. alternating to average power ratio=20), receptively, at a frequency of $15 \mathrm{~Hz}$ with a constant speed of $2000 \mathrm{rpm}$. The set value of inner rotor speed is $4000 \mathrm{rpm}$ in this case which is chosen to be close to the efficient speed operation of the system.

Fig. 3 shows the above mentioned mechanical load torque applied to the outer rotor of EVT and also the speed of outer rotor which shows a good tracking performance with maximum error of $\pm 3.5 \%$. Furthermore, Fig. 4 shows the evolution of the consumed power from three phase grid supply with time, $P_{\text {grid }}$ with an average found to be $2.61 \mathrm{~kW}$. It is clear from this figure, that the developed energy management approach is capable of minimizing the grid energy which only accounts for the active power $\left(P_{\text {active }}=2 \mathrm{~kW}\right)$ and internal EVT losses. The oscillating energy is stored in the inner rotor of the EVT and supplied back to drive the load when required. There is a limited energy exchange between the capacitor at DC bus and the EVT which can be seen in the evolution of input electrical energy to EVT from grid supply, $P_{e, i n}$ when compared to $P_{\text {grid }}$. Energy cost is calculated based on the average active power drawn from the grid with the associated fundamental power factor, which gives $€ 5227$ for a total amortization period of 5 years.

Fig. 5(a) shows the supply voltage and the resulting phase current. It is clear from this figure that the fundamental power factor is high, at 0.95 . However the total power factor is a bit
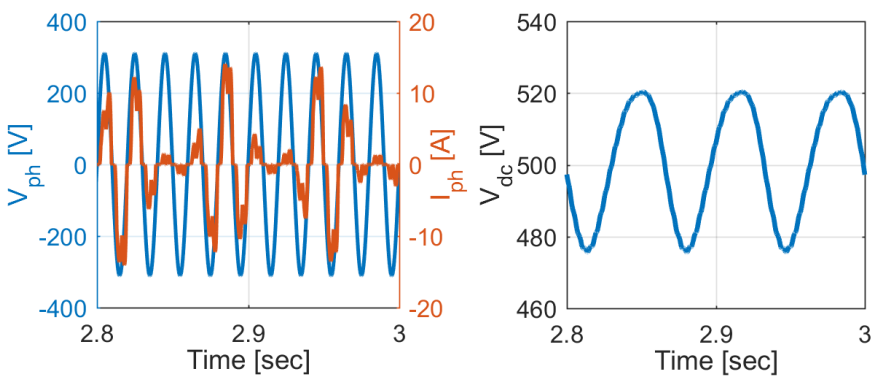

Fig. 5. (a) Supply Voltage and current waveforms. (b) Voltage at the DC bus.

lower, but still reasonable at 0.7 , which can be improved by adding extra line inductance to the system as it is usual in PFEbased systems, see [2]. Fundamental power factor is used to compute the energy cost, i.e. the energy cost is penalized if the fundamental power factor is lower than a specific value, e.g. 0.9. On the other hand, the total power factor is an indication about the system total harmonic distortion (THD), i.e. higher the total power factor, lower the THD. Total power factor is often a parameter that needs to be taken into account in the design phase [2]. Moreover, Fig. 5(b) shows the DC voltage variation with time which respects the constraint limit given by (11). It is obvious here that the voltage ripple is small, which means that very limited energy is stored in the capacitance at the DC-bus. Also, lower voltage ripple results in lower inverter loss and the associated iron loss due to the change in the modulation of the pulse width of the inverter driver circuits [11].

A trade-off between the load speed tracking and the energy costs is observed with the current topology. The tracking performance can be improved to $\pm 1 \%$ maximum error but the costs are increased by $17 \%$. The reason mainly lies in the estimation of current dynamics for higher electromagnetic torque generation requirements during dynamic load variations as explained in section II-A. Average of the power consumed from grid $\left(P_{\text {grid }}\right)$ rose by $17 \%$ to compensate for the increased losses with increased currents through the windings. Hence the choice of trade-off depends on the application and its requirements on how much tolerance can be given in terms of the performance and costs.

\section{Conclusions}

In this paper, the dynamic capability of an EVT has been analyzed through successful implementation of an energy management strategy on an industrial drivetrain topology. The control methodology minimizes the energy consumed from grid supply in such a way that grid energy is used to compensate only for the DC average power and internal EVT losses. The high frequency oscillating load is driven solely by a mechanical energy storage component by storing and supplying back the energy during acceleration and deceleration of load. The EVT is shown to be an energy storage component in itself, however an extra flywheel can always be added to its inner rotor depending of energy storage requirements. The 
developed control strategy shows a good tracking performance of load speed for up to $\pm 1 \%$ error, however with a trade-off with overall energy costs.

\section{ACKNOWLEDGMENT}

This work is performed as part of the EMTECHNO-SBO project, funded by the Institute for the Promotion of Innovation through Science and Technology in Flanders (VLAIO) and Flanders Make, the strategic research centre for the manufacturing industry in Belgium.

\section{REFERENCES}

[1] E. Vinot and R. Trigui, "Optimal energy management of HEVs with hybrid storage system," Energy Conversion and Management, vol. 76, pp. 437-52, 2013.

[2] B. Lenaerts, A. Abdallh, D. Maes, B. Mrak, T. Galle, and W. Dewaele, "Total cost of ownership optimization of manufacturing machines with fast energy storage," Proceeding of the $18^{t h}$ IEEE International Conference on Power Electronics and Motion Control (IEEE-PEMC), Budapest, Hungary, 26-30 August, 2018.

[3] E. Vinot, R. Trigui, Y. Cheng, C. Espanet, A. Bouscayrol, and V. Reinbold, "Improvement of an EVT-based HEV using dynamic programming, IEEE Trans. Veh. Technol., vol. 63, no.1, pp. 40-50, 2014.

[4] A. Abdelsalam and S. Cui, "A fuzzy logic global power management strategy for hybrid electric vehicles based on a permanent magnet electric variable transmission," Energies, vol. 5, pp. 1175-119, 2012.
[5] Q. Xu, S. Cui, L. Song, and Q. Zhang, "Research on the power management strategy of hybrid electric vehicles based on electric variable transmissions," Energies, vol. 7, pp. 934-60, 2014.

[6] M. Vafaeipour, M. El Baghdadi, F. Verbelen, P. Sergeant, J. Van Mierlo, K. Stockman, and O. Hegazy, "Technical assessment of utilizing an electrical variable transmission system in hybrid electric vehicle," Proceeding of the IEEE Transportation Electrification Conference and Expo, Asia-Pacific (ITEC Asia-Pacific), Bangkok, Thailand, 6-9 June, 2018.

[7] Y. Zhu, M. Cheng, and H. Zang, "Sensorless control for the EVT-based new dual power flow wind energy conversion system," Energies, vol. 10, 888, 2017.

[8] J. Druant, H. Vansompel, F. De Belie, J. Melkebeek, and P. Sergeant, "Torque analysis on a double rotor electrical variable transmission with hybrid excitation," IEEE Transactions on Industrial Electronics, vol. 64, no. 1 , pp. $60-68,2017$.

[9] J. Druant, H. Vansompel, F. De Belie, and P. Sergeant, "Optimal control for a hybrid excited dual mechanical port electric machine," IEEE Transactions on Energy Conversion, vol. 32, no. 2, pp. 599-607, 2017.

[10] J. Druant, H. Vansompel, F. De Belie, and P. Sergeant, "Loss identification in a double rotor electrical variable transmission," IEEE Transactions on industrial electronics, vol. 64, no. 10, pp. 7731-7740, 2017.

[11] P. Rasilo, A. Salem, A. Abdallh, F. De Belie, L. Dupré, and J. Melkebeek, "Effect of multilevel inverter supply on core losses in magnetic materials and electrical Machines," IEEE Transactions on Energy Conversion, vol. 30, no. 2, pp. 736-744, 2015. 